\title{
Apoptosis and Progression of Hepatic Fibrosis in Hepatitis C Patients
}

\author{
Maria Isabel Schinoni, Raymundo Paraná \\ and Daniel Cavalcante
}

\author{
Edgard Santos University Hospital; Salvador, BA, Brazil
}

\begin{abstract}
Hepatitis $\mathrm{C}$ is a worldwide endemic disease, affecting roughly 200 million people. It has a variable prognosis, depending on the progression to fibrosis. During the last five years, the importance of apoptosis for the pathogenesis of various diseases, including hepatitis, has been recognized. It has been suggested that an increase in $T$ cell-apoptosis during a hepatitis $C$ virus infection is the cause of impaired regulation of the immune cellular response, helping to maintain infection. Thus, the interest in discovering the probable mechanisms by which the hepatitis $\mathbf{C}$ virus perpetuates in the liver, and to determine the conditions that predispose for progression of this disease, makes investigation of apoptosis in hepatic injury of great interest. We have made an overview of the various mechanisms by which the cell, more specifically the hepatic cell, is affected by apoptosis, and how it interacts with the hepatitis $C$ virus and the immune system.
\end{abstract}

Key Words: Apoptosis, hepatitis C, liver fibrosis.

Hepatitis $\mathrm{C}$ is a worldwide endemic disease, affecting roughly 200 million people. It has a variable prognosis, depending on the progression to fibrosis. Over the past years, apoptosis (programmed cellular death) and molecules that could stimulate or inhibit this process, such as the interleukins, have received considerable attention in studies of hepatitis $\mathrm{C}$.

Recent studies have shown that the physiological process of apoptosis can be transformed into a pathological process, which can stimulate hepatic fibrosis in hepatitis $\mathrm{C}$ or can contribute to treatment failure.

Over the last five years, the importance of apoptosis for the pathogenesis of various diseases has been extensively investigated. Apoptosis is a Greek term that means "the fall of the old leaves of the autumn trees". This term describes the process by which undesirable, damaged or old cells are eliminated from multicellular organisms. Kerr reported on this phenomenon in the liver in 1970, in a study that recognized organ atrophy after ligature of the portal vein, followed by a process of cellular condensation, fragmentation and separation of neighboring cells [1].

Through the apoptotic process, the cells shrink and become separated from each other. An invagination of the cytoplasm becomes a vesicle and engulfs intact organelles. At the same time, the nucleus is fragmented. The disintegrated cell and its membranes are called apoptotic bodies, which are phagocytized by neighboring cells or by specialized phagocytes, such as Kupffer cells in the liver.

Apoptosis differs from cellular necrosis, because it is actively controlled and the membrane integrity is maintained,

Received on 23 September 2005; revised 14 February 2006.

Address for correspondence: Dr. Dr. Maria Isabel Schinoni . Av. Juracy Magalhães J. 2096 Sala 510, Salvador- Bahia. 4192.000 Brazil. Fax: 55-71-21084651. Phone: 55- 71-32642064.

E-mail; misabelschinoni@terra.com.br.

The Brazilian Journal of Infectious Diseases 2006;10(2):117-121. (C) 2006 by The Brazilian Journal of Infectious Diseases and Contexto Publishing. All rights reserved. avoiding extravasation of intracellular material and an inflammatory response [2]. It has been suggested that an increase of $\mathrm{T}$ cell-apoptosis during viral hepatitis $\mathrm{C}$ causes impaired regulation of the immune cellular response and maintenance of infection [3]. Thus, in order to discover the probable mechanisms by which the hepatitis $\mathrm{C}$ virus perpetuates in the liver, and to evaluate the conditions that predispose for its progression, apoptosis in hepatic injury should be investigated.

\section{Physiopathogeny of Apoptosis}

Two mechanisms by which the apoptotic process can occur have been described: 1 . death receptor (DR) or extrinsic mechanism, and 2. mitochondrial or intrinsic mechanism. The former involves the binding and/or oligomerization of cell surface receptors that trigger apoptosis. Hepatic cells probably express DRs because of pressure to eradicate the hepatotropic viruses. Immunocytes use apoptosis to eradicate the infected cells. In contrast, the mitochondrial process is triggered by various types of responses to intracellular stress, including DNA damage, changes in intracellular calcium concentration and endoplasmic stress. Both mechanisms can be present simultaneously [4].

Mitochondrial dysfunction often plays a critical role in augmenting the apoptotic process and in integrating death receptor-initiated and stress signals into a common final pathway $[5,6]$. Mitochondrial release of cytochrome $\mathrm{C}$ is a near-universal event in apoptosis; it triggers a final, caspase-dependentapoptosis cascade, culminating in cellular fragmentation. [7]

The death receptors on the cellular surface belong to a cytokine superfamily, tumor necrosis factor or nerve growth factor (TNF/NGF). These receptors are expressed in many tissues and cells, including hepatic tissue. Apoptosis mediated by these receptors has a major role in a variety of biological processes, such as tissue injury, immunologic and lymphocytic homeostasis, and protection against pathogenic microorganisms [5]. 


\section{Death Receptors}

Six types of DRs have been identified, but only the following are considered to play a role on liver apoptosis: 1 . Fas (CD95), 2. Tumor necrosis factor receptor (TNF_R1), 3. Receptor-1 binding inducing TNF (TRAIL-R1), also referred to as death receptor 4 (DR4), 4. Receptor-2 binding inducing TNF (TRAIL-R2), also called death receptor-5 (DR5). The endoplasmic portion of these polypeptides contains a sequence between amino acids 60 and 80, which is referred to as a death domain, which binds to specific adapting proteins that transmit a code to connect and activate caspase, a cysteine protease that triggers the apoptosis cascade. $[1,4,6,9,10]$

Fas. Fas is a cellular surface protein with a molecular weight of 42-52 kDA. It promotes apoptosis after it binds to an inactivated binding (FasL), a transmembrane protein with a homotrimeric structure that is expressed on the cellular surface of active $\mathrm{T}$ cells and NK cells, working as an effector of these cytotoxic cells to remove virus-infected cancerous cells. Misfunction of the Fas system leads to hyperplasia of lymphoid organs and liver, accelerating auto-immune diseases and tumor genesis, while the exacerbation of this system leads to tissue destruction [2]. FasL expression has been found to be increased in liver infiltrating T lymphocytes. FasL release from these activated T cells may initiate the apoptotic death signal in Fas-bearing hepatocytes and in pro-inflammatory-activated cells that continuously migrate from extra-hepatic sites. [11]

TNF- $\alpha$. TNF- $\alpha$ is pleiotropic cytokine expressed as a type II integral transmembrane protein with a homotrimeric structure. It is found mainly in macrophages, including Kupffer cells, monocytes and T-cells, in response to infections and inflammatory conditions, as well as in B-cells, fibroblasts and hepatocytes. It binds to two different receptors: TNF-R1 and TNF-R2; but only the former has an intracellular death domain, being the only mediator of apoptotic signal related to this cytokine.

Trail Receptor. TRAIL/APO-2 is another type II transmembrane protein that can turn into a soluble form by cleavage. The biologically active form is a homotrimer, with cysteine residues at position 230, coordinating an essential zinc ion for association with the trimer and its own activation. This is capable of binding to five different receptors: TRAIL-R1, TRAIL-R2, TRAIL-R3 (also called TRID), TRAIL-R4 and the soluble osteoprotegerin receptor (OPG). Among these, only TRAIL-R1 and TRAIL-R2 contain an essential endoplasmic death domain to translate the apoptotic signal. [1,4].

\section{Mechanism of Apoptosis Signalization by Death Receptors}

When ligand/binding Fas (FasL) binds to Fas, and TNF binds to TNF-R1, the individual molecules trimerize and aggregate, leading to the union of the death domains.
Rearrangement allows the Fas domain to become linked to an adaptor present in the cytoplasm, originating a Fas associated death domain (FADD) or a TNF receptor-associated death domain (TRADD), which binds to FADD. Finally, it binds to an endoplasmic zymogen, known as procaspase 8 , transformed into caspase 8 through proteolytic cleavage [1]. This is a proteolytic enzyme synthesized as an inactive proenzyme, and when proteolytically processed it constitutes an active complex that initiates an intracellular cascade that amplifies apoptotic signaling. It has been demonstrated that caspase plays an important role in many hepatic diseases $[6,10]$.

In a study conducted by Bantel et al. [9] , the activation of caspase was found to be promoted by anti-caspase antibodies on hepatic biopsy; they compared it with the degree of histological inflammatory activity, finding that $\mathrm{HCV}$-induced hepatic lesions are characterized by an increase in caspase activation and that the degree of necroinflammatory activity is correlated with the activation of this enzyme. Patients with grade I hepatitis revealed activation of caspase 3 and 7 in $7.7 \%$ and $8.7 \%$ of hepatocytes, respectively, while patients with grade III had $20.9 \%$ and $19.5 \%$. There was no correlation with other disease markers, such as viral load or transaminase levels, which shows that chronic damage and hepatocyte loss can occur in HCV-infected patients without biochemical manifestations, and that progression of the disease can occur with normal levels of transaminases. This positive correlation between activation of caspase and inflammatory damage in $\mathrm{HCV}$ infection would be useful for the development and interpretation of new therapeutic agents.

In another study, conducted by McPartland et al. [12] , hepatocyte apoptosis was assessed in liver tissue from 32 patients with chronic hepatitis B or C. Apoptosis rates were measured using H\&E morphological assessment and immunohistochemical staining with antibodies to activated caspase-3 and M30. Histological necro-inflammatory activity was scored using the Knodell scoring system. It was found that apoptotic scores obtained with an antibody to activated3 are significantly higher in cases of chronic viral hepatitis with greater histological necro-inflammatory scores, supporting a central role for apoptosis in disease pathogenesis.

The Fas complex (FADD) plus caspase 8 is referred to as the death inducing signaling complex (DISC). Activation of caspase initiator catalyzes a series of proteolytic events that contribute to the process of apoptosis $[1,4]$. It has been demonstrated that blocking of this complex can lead to inhibition of apoptosis [8].

TNF- $\alpha$ is produced by macrophages, including Kupffer cells, monocytes and T-cells, as well as B-cells, fibroblasts and hepatocytes. When TNF-R1 is linked to TNF, it trimerizes, allowing the formation of TRADD, which associates with a death domain and serves as a platform for the recruitment of various molecules, including FADD, TNF-R (TRAF-2) and RIP. The union of RIP and TRAF-2 promotes activation of 
transcription factors that lead to the activation of genes that oppose apoptosis. The mechanism is: when RIP and TRAF bind to death receptors, a nuclear factor protein kinase, NF$\kappa \beta$ (NIK), is activated; this in-turn activates the catalytic complex, Iк $\beta$ kinase (IKK complex), leading to phosphorylation of the inhibitory protein of NF- $\kappa \beta$, known as I $\kappa \beta \alpha$; then it is degraded, allowing NF- $\kappa \beta$ to translocate to the nucleus and initiate transcription of target genes that are involved in survival pathways [4].

Undoubtedly, inhibition of NF- $\kappa \beta$ sensibilizes apoptotic cells [4]. This transcriptional factor, $N F-\kappa \beta$, is required for the activation of lymphocytes, and for the proliferation and expression of various cytokines. It is rapidly activated by a large spectrum of microbial and viral pathogens, perpetuating progression of cellular cycles and then protecting against apoptosis.

Apoptosis inhibition was found to be mediated by Fas and TNF- $\alpha$ in hepatoma cells at stage G2 (HepG2), through activation of NF- $\kappa \beta$ by proteins from the HCV core [13]. Activation of $\mathrm{NF}-\kappa \beta$ appears to be responsible for the resistance of the hepatic cells to express structural proteins of $\mathrm{C}$ virus in response to TNF $\alpha$ [14]. Opposing this hypothesis, another study showed that a protein from the HCV core would not inhibit apoptosis that is induced by Fas or TNF $\alpha$ [15]. There is considerable evidence of both pro- and anti-apoptosis effects of HCV proteins. To avoid this bias, Kalkeri et al. [16] used hepatoma cells (Hep G2) transfected with the complete virion to check for the expression of structural and nonstructural proteins of $\mathrm{HCV}$; they demonstrated that the expression of such proteins is toxic to the cell and promotes apoptosis.

\section{Apoptosis of Hepatocyties}

Hepatocytes express Fas, TNF-R1, TRAIL-R1, and TRAIL$\mathrm{R} 2$. The role of Fas and TNF-R1 in hepatic apoptosis is well known, based on the hepatotoxicity caused by the agonist antibodies anti-Fas and TNF $\alpha$, both in vitro and in vivo [17]. However, the hepatotoxic potential of TRAIL is controversial. It seems that it plays a less significant role in the progression of apoptosis, though its expression appears to be induced by DNA damage [4].

\section{Apoptosis in Hepatic Stellate Cells (HSC)}

The hepatic stellate cell is a resident perisinusoidal mesenchymal cell that is a central effector of fibrosis; it is part of the final common pathway of the wound-healing response of the liver, playing an active role in hepatic inflammation. These cells migrate in response to cytokines released by monocytes, and they secrete a number of pro-inflammatory cytokines and chemokines that participate in the activation of white blood cells, thus amplifying the inflammatory response. Stellate cells are usually inactivated, but with appropriate stimuli they are activated and change their pattern of genotypic and phenotypic expression, undergoing a myofibroblastic transdifferentiation or activation, leading to an accumulation of extracellular matrix. These changes in cellular function are analogous to those found in T- and dendritic cells. Probably the sensitivity of HSC to apoptosis mediated by DR also changes, depending on whether it is in a quiescent or activated form. HSC cultures resist apoptosis mediated by Fas; however, when these cells are activated, they express Fas and become susceptible to apoptosis by TRAIL [4].

\section{Viral Hepatitis}

$\mathrm{HBV}$ and $\mathrm{HCV}$ are the main etiological agents of viral hepatitis. T-cytotoxic lymphocytes (CTLs) recognize viral epytopes after intracellular processing and expression on the hepatocyte surface in conjugation with MHC I. CTLs induce tissue destruction, which is the most visible signal of viral hepatitis lesion. Apoptosis has a significant role in viral hepatitis. Apoptotic bodies, previously referred to as Councilman bodies or acidophilus bodies, are present in patients with viral hepatitis [4].

Apoptosis induction in infected cells is a defense mechanism to limit viral replication and promote their elimination. Various pathways are implicated in this process, including Fas and TNF $\alpha$ systems, as well as perforin/granzime [2].

Fas concentrations are correlated with viral hepatitis activity. Cytotoxic lymphocytes express Fas and induce hepatic apoptosis through signaling. Fas expression can be induced by viral proteins $[20,21]$.

Mita et al. (1997) immunohistochemically assessed Fas antigen expression in hepatitis $\mathrm{B}$ and $\mathrm{C}$, to determine if it was correlated with the severity of inflammation [18]. FasL expression was detected in liver mononuclear cell infiltrates of patients with chronic HCV, suggesting an important role of the Fas system in hepatic lesions induced by this virus.

In another study, transgenic mice which expressed HCV core protein (the first protein expressed in the initial phase of viral infection) to $\mathrm{T}$ lymphocytes, had little response of $\mathrm{T}$ lymphocytes, including interferon gama and interleukine-2 production [19]. In comparison with non-transgenic mice, the reduction of response may be due to increased susceptibility of the T-lymphocytes to Fas-mediated apoptosis. HCV proteins can stimulate or inhibit Fas apoptosis through mechanisms that normally maintain viral infection [20-22]. Elevated Fas expression and excessive apoptosis are characteristic of fulminant hepatitis. After experimental induction of apoptosis with a Fas-agonist, mice developed fulminant hepatic failure, with massive necrosis and inflammation [2].

Enhanced Fas-mediated hepatocyte apoptosis has been well documented in HCV-and HBV-associated chronic liver diseases; Fas expression increases in parallel with chronic 
hepatitis $\mathrm{C}$ progression, demonstrating the important role of cell death $[11,23]$. Infected hepatocytes exhibit enhanced Fas expression and develop increased susceptibility to FasLmediated apoptosis. Thus the Fas- FasL pathway plays an important role in liver cell injury $[11,24,25]$. The HCV core protein, a structural component of the virus, may have a regulatory function, as it can enhance or inhibit apoptosis, depending on experimental conditions and on the type of cell used; also, both the NS3 and NS5A proteins initially were found to have anti-apoptotic effects; but new studies have demonstrated that they also have pro-apoptotic action $[9,10,11,25]$.

TNF-R1 expression is increased in HBV and HCV, and TNF$\alpha$ production is increased in peripheral mononuclear cells. HCV core protein can bind to the TNF-R1 endoplasmic domain; this interaction promotes apoptosis in mouse and human cell lineages. However, TNF-R1 signaling is extremely complex, leading to apoptotic as well as survival signals. HCV core protein also activates NF- $\beta \beta$ through TNF-R1 $[4,9]$.

\section{Apoptosis and Liver Fibrosis}

As apoptosis is a first step in hepatic lesions, and fibrosis is a final response of HSC to this process, there may be a direct relationship between these two processes. Fibrosis is one of the best parameters for correct evaluation of this disease, because it is correlated with cirrhotic evolution. Apoptosis results in the formation of apoptotic bodies, which are cleared from tissues by phagocytosis. This is necessary to protect the tissue from damage caused by the liberation of pro-inflammatory material. The macrophages that engulf the apoptotic bodies in vitro inhibit pro-inflammatory cytokine production, through mechanisms that involve TGF- $\beta$ secretion [26]. Epithelial cells and fibroblasts may participate in this process. Stellar cells are close to hepatocytes, in a strategic location to phagocyte apoptotic bodies generated by dead hepatocytes. If TGF- $\beta$ production is increased in HSC, a pro-fibrogenic response is generated in the liver. HSC cells englobe apoptotic bodies and incubation with them stimulates TGF- $\beta$ expression and collagen Ia mRNA [27]. These observations strongly suggest that the phagocytotic processing by HSC may link hepatic lesion and fibrosis. Soon after hepatic damage, HSCs go through morphological and phenotypical modifications, resulting in their activation and collagen matrix deposition. Soon after activation, HSCs exhibit apoptosis $[6,11]$.

A direct link between accumulation of inflammatory mediators and apoptosis has been reported. Upregulated apoptosis under pathological conditions can disrupt hepatocyte integrity; when the magnitude of apoptosis overcomes the capacity to clear cellular debris, apoptosis bodies undergo spontaneous disruption and release their contents. A direct link between accumulation of inflammatory mediators and apoptosis has been found. It was also demonstrated that hepatocyte apoptosis is strong stimulus for neutrophil extravasation via receptor death. Finally, engulfment of neutrophil apoptotic bodies by macrophages and/or Kupffer cells can induce the expression of death ligands [6].

\section{Study of Apoptosis Mediation in the Liver}

The methods to identify apoptotic hepatocytes are limited because they are quickly englobed by adjacent macrophages. The simplest method consists of manual counts of these macrophages, which are stained with hematoxilin and eosin. Using this method, Shimamatsu \& Wanless [28] identified two types of apoptotic bodies in the liver: spherical acidophilic bodies located away from adjacent hepatocytes (Councilman bodies) and star-shaped acidophilic bodies, which are retracted but still united to hepatocytes. The apoptotic body nucleus can be pycnotic, fragmented or absent; phagocytosed apoptotic bodies, and cells exhibiting condensation of chromatin as an initial manifestation of apoptosis have also been described [21-28].

In another study [29], acidophilic bodies were quantified in liver biopsies from patients with recurrent HCV infection after liver transplant, in patients with non-complicated graft rejection due to other etiologies (non-HCV), and in patients carrying non-transplanted HCV. A doubling in the proportion of acidophilic bodies at the initial stage was found in the group with recurrent $\mathrm{HCV}$ infection after grafting, when compared to the other groups, showing the importance of an evaluation of apoptosis for differential diagnosis between reinfection and rejection [22-29].

TUNEL is another technique that demonstrates DNA cleavage in situ. During apoptosis, DNA cleavage leads to an increase in the number of hydroxyl-free terminals. The indication of the transferring terminal of deoxynucleotide mediating deoxiuridine triphosphate (dUTP) is named the TUNEL technique. It has the inconvenience of confusion between necrosis and apoptosis, and it depends on the technique used to mark the apoptotic cells.

Fluorescent microscopy can detect apoptosis in isolated cells and in tissues. Fluorescein binding (DAPI) is used to detect nuclear changes, showing changes in condensation and margination of chromatin and in nuclear fragmentation. It can also be measured by flux cytometry, using nucleic acids bound to a fluorescent marker.

Anexin V. Anexin V is another technique used to detect apoptosis. Phosphatidilserin, an enzyme externalized on plasmatic membrane at the initial phase of apoptosis is identified. It has the disadvantage of being a non-quantitative method [2]. There are a variety of markers available to measure apoptosis. For material fixed in formalin, the best method is Apoptag in situ, in Apoptosis Detection kits that detect DNA fragmentation through TUNEL (which associates fragmented DNA with the definitive morphology of cellular apoptosis). 
Caspase Study. Caspase is a family of enzymes that begin the apoptosis cascade; these are secreted as caspase 8 and 3 zymogens and are activated in sequence, such as caspase 8 and 9, which are initiator caspases that trigger activation of downstream effector caspases, including caspase 3, 6 and 7 . Inmunohistochemical procedures allow study of the different caspases, mainly caspase 3 , identifying apopotic cells and apoptotic rates at an early stage of apoptosis. [6,12]

\section{References}

1. Kaplowitz N. Hepatology: A century of progress. Cell death at the millennium. Clinics in Liver Disease 2000;4(1):25768.

2. Patel T. Apoptosis in hepatic pathophysiology. Clinics in Liver Diseases 2000;4(2):295-317.

3. Nelson D. The Immunopathogenesis of Hepatitis C Virus Infection. Clinics in Liver Disease 2001;5(4):931-53.

4. Yoon JH., Gores G. Death receptor-mediated apoptosis and the liver. Journal of Hepatology 2002;37:400-10.

5. Hengartner MO. The biochemistry of apoptosis. Nature 2000;407;770-6.

6. Canbay A., Friedman S., Gores G. Apoptosis: The nexus of liver injury and Fibrosis Hepatology 2004; Vol.39(2).

7. Green DR, Reed JC. Mitochondria and apoptosis. Science 1998;281:1309-12.

8. Seino K., Setoguchi Y., Ogino T., et al. Protection against Fasmediated and tumor necrosis factor receptor 1-mediated liver injury by blockade of FADD without loss of nuclear factorkappaB activation. Annals of Surgery 2001;234(5):681-8 .

9. Bantel H., Lügering A., Poremba CH., et al. Caspase activation correlates with the degree of inflammatory liver injury in Chronic Hepatitis C Virus infection. Hepatology 2001;34(4):758-67.

10. Prikod 'ko E., Prikod'ko G., Siegel R., et al. The NS3 protein of hepatitis $\mathrm{C}$ virus induces caspase-8-mediated apoptosis independent of its proteases or helicases activities. Virology, 2004;329:53-67.

11. Kountouras J., Zavos C., Chatzopoulos D. Apoptosis in Hepatitis C. Journal of Viral Hepatitis, 2003; Vol.10:33542.

12. Mc Partland J., Guzail M., Kendall C., Pringle J. Apoptosis in chronic viral hepatitis parallels histological activity. International Journal of Experimental Pathology 2005;86:1924.

13. Marusawa H., Hijikata M., Chiba T., Shimotohno K. Hepatitis $\mathrm{C}$ virus core protein inhibits fas- and tumor necrosis factor a-mediated apoptosis via NF-kB activation. Journal of Virology 1999;73:4713-20.

14. Tai D., Tsai S L., Chen Y., et al. Activation of nuclear factor kB in hepatitis $\mathrm{C}$ virus infection: implications for pathogenesis and hepatocarcinogenesis. Hepatology 2000;31:656-64.
15. Dumoulin F., Van dem Bussche., Sonhe J., et al. Hepatitis C virus core protein does not inhibit apoptosis in human hepatoma cells. European Journal of Clinical Investigation 1999;29:940-6.

16. Kalkeri G., Khalap N., Garry R., et al. Hepatitis C Virus Protein Expression Induces Apoptosis in Hep G2 Cells. Virology 2001;282:26-37.

17. Ogasawara J., Watanabe-Fukunaga R., Adachi M., et al. Lethal effect of the anti-Fas antibody in mice. Nature 1993;364:806-9.

18. Mita E. Apoptosis in human diseases: role of Fas system in liver cell injury by viral hepatitis Rinsho Byori 1997;45(5):477-82.

19. Soguero C., Joo M., Chianese-Bullock KA., et al . Hepatitis C virus core protein leads to immune suppression and liver damage in a transgenic murine model. Journal of Virology 2002;76(18):9345-54.

20. Okazaki M., Hino K., Fuji K., et al. Hepatic Fas antigen expression before and after interferon therapy in patients with chronic hepatitis C. Digestive Diseases and Science 1996; 41:2453-58.

21. Ray RB., Meyer K., Ray R. Suppression of apoptotic cell death by hepatitis $\mathrm{C}$ virus core protein. Virology 1996;226:176-82.

22. Honda M., Kaneko S., Matshubita E., et al. Hepatitis C virus core protein induces apoptosis and impairs cell-cycle regulation in stably transformed Chinese hamster. Hepatology 2002;31:1351-9.

23. Jarmay K, Karacsony G., Ozvar Z., et al. Assessment of histological features in chronic hepatitis $\mathrm{C}$ Hepatogastroenterology 2002;49:239-43.

24. Toubi E., Kessel A., Goldstein L., et al. Enhanced peripheral Tcell apoptosis in chronic $\mathrm{C}$ virus infection associated with liver disease severity. Journal of Hepatology 2001; 35:77480.

25. Calabrese F., Pontisso P., Pettenazo E., et al. Liver cell apoptosis in chronic hepatitis $\mathrm{C}$ correlates with histological but not biochemical activity or serum HCV-RNA levels. Hepatology. 2000;31:1153-9.

26. Fadok V.A., Bratton D.L., Konowal A.,.et al. Macrophages that have ingested apoptotic cells in vitro inhibit proinflammatory cytokine production through autocrine/ paracrine mechanisms involving TFG-beta, PGE2, and PAF. Journal of Clinical Invesigation 1998; 101:890-8.

27. Fadok V.A.., Bratton D.L., Henson P.M. Phagocyte receptors for apoptotic cells: recognition, uptake, and consequences. $\mathrm{J}$ Clin Invest 2001;108(7):957-62.

28. Shimamatsu K.,Wanless I.R. Role of ischemia in causing apoptosis, atrophy and nodular hyperplasia in human liver. Hepatology 1997;26:343-50.

29. Saxena R., Crawford J., Navarro V., et al. Utilization of Acidophil bodies in the diagnosis of recurrent hepatitis $\mathrm{C}$ infection after orthotopic liver transplantation. Modern Pathology 2002;15(9):897-903. 